\title{
Nueva forma anatómica de cara oclusal en primeros molares superiores permanentes
}

\author{
Gabriela G. Bessone*, Esp. ${ }_{1}$, María A. Guiglioni, Esp. María M. González, Ph.D. ${ }_{2}$ \\ ${ }_{1}$ Departamento de Ciencias Básicas \\ ${ }_{2}$ Departamento de Clínicas Integradas, Facultad de Odontología \\ Universidad Nacional del Nordeste, Corrientes, Argentina
}

Recibido: 9 de diciembre del 2013. Aprobado: 27 de marzo del 2014.

*Autor de correspondencia: Gabriela Guadalupe Bessone, Facultad de Odontología, unNE Corrientes, Argentina. Av. Libertad 5.450 Corrientes, teléfono: 3794 350079, correo electrónico: gbessone@odn.unne.edu.ar

Cómo citar este artículo: Bessone GG, Guiglioni MA, González MM. Nueva forma anatómica de cara oclusal en primeros molares superiores permanentes. Rev Nac Odontol. 2014;10(18):15-20. doi: http://dx.doi.org/10.16925/od.v10i18.717

Resumen. Introducción: un surco no tipificado, en los primeros molares superiores permanentes (PMSP), permitió distinguir dos tipos de cara oclusal: la descrita por Figún y Garino, Tipo I para el grupo de investigación, y Tipo II, caracterizada por la presencia de un surco sobre la apófisis oblicua, que la discurre sin interrumpirla. Este elemento dio lugar a la realización de estudios con el fin de determinar su presencia e investigar origen, dirección y longitud para caracterizarlo y registrarlo como una nueva forma anatómica de cara oclusal. Métodos: se trabajó con PMSP naturales inertes y de pacientes para evidenciar la presencia del surco y con PMSP que presenten el surco para caracterizarlo. El método fue observacional y descriptivo. Resultados: existe un predominio de casos en los que la cara oclusal del PMSP presenta un surco que, sin cortar a la apófisis oblicua, la recorre parcialmente. Se origina en la fosa principal central triangular. La dirección es hacia distal y palatino y su longitud varía entre 1 y $2 \mathrm{~mm}$. Esto ha permitido registrar esta cara oclusal, como PMSP Tipo II. Conclusión: el PMSP presenta dos tipos de cara oclusal: Tipo I y Tipo II.

Palabras clave: diente molar, anatomía, dentición permanente, corona del diente.

New anatomical form of occlusal surface on permanent maxillary first molars

Abstract. Introduction: an undefined sulcus on the permanent maxillary first molars (PMFM) allowed the identification of two types of occlusal surfaces: that described by Figún and Garino, Type 1 for the research group, and Type 2, characterized by the presence of a sulcus above the oblique ridge, that runs along it without breaking it. This observation allowed studies to be undertaken in order to determine its presence and investigate its origin, direction and length, to characterize it and register it as a new form of occlusal surface. Methods: we worked with natural PMFM, both inert and in patients, to demonstrate the presence of the sulcus and the Pмғм that present the groove in order to characterize it. The method was observational and descriptive. Results: there is a predominance of cases in which the occlusal surface of the PMFM presents a sulcus that partially runs along the oblique ridge, without breaking it. It begins in the main central triangular fossa and runs towards the distal and palatal, its length varying between 1 and $2 \mathrm{~mm}$. These results have allowed this occlusal surface to be registered as Type II PMFM. Conclusion: the PMFм presents two types of occlusal surface: Type I and Type II.

Keywords: molar tooth, anatomy, permanent dentition, tooth crown.

\section{Nova forma anatômica de face oclusal em primeiros molares superiores permanentes}

Resumo. Introdução: um sulco não tipificado, nos primeiros molares superiores permanentes (PMSP), permitiu diferenciar dois tipos de face oclusal: a descrita por Figún e Garino, Tipo i para o grupo de pesquisa, e Tipo II, caracterizada pela presença de um sulco sobre a apófise oblíqua, que se estende sem interrupção. Este elemento levou à realização de estudos com o objetivo de determinar sua presença e pesquisar a origem, direção e longitude para caracterizá-lo e registrá-lo como uma nova forma anatômica de fase oclusal. Métodos: trabalhou-se com PMSP naturais inertes e de pacientes para evidenciar a presença do sulco e PMSP que apresentem o sulco para caracterizá-lo. O método foi observacional e descritivo. Resultados: existe um predomínio de casos em que a face oclusal do PMSP apresenta um sulco que, sem cortar a apófise oblíqua, estende-se parcialmente por ela. Origina-se na fossa principal central triangular. A direção é no sentido distal e palatino e sua longitude varia entre 1 a $2 \mathrm{~mm}$. Isso permitiu registrar essa face oclusal, como PMSP Tipo II. Conclusão: o PMSP apresenta dois tipos de face oclusal: Tipo I e Tipo II.

Palavras-chave: Dente molar, anatomia, dentição permanente, coroa do dente. 


\section{Introducción}

El primer molar superior permanente (PMSP) es la primera pieza dentaria permanente que aparece en la cavidad bucal a los 6 años de edad, en condiciones normales. Como toda pieza dentaria, posee dentro de su conformación externa diversos elementos arquitectónicos que le confieren características propias y que le permiten, a su vez, diferenciarse del resto de las piezas dentarias presentes en boca [1-5].

El PMSP presenta una cara oclusal o superficie triturante, de forma romboidal, con cuatro cúspides, dos hacia vestibular y dos hacia palatino. Puede observarse que estas se encuentran separadas por dos fosas, una central de donde parten dos surcos principales: uno hacia vestibular y otro hacia el reborde marginal mesial. Ambos surcos forman un ángulo aproximado de $95^{\circ}$, donde queda delimitada la cúspide mesiovestibular, que se menciona de acuerdo con su tamaño en segundo orden [2].

La segunda fosa principal descrita por los diferentes autores es la llamada fosa distal. De ella parten dos surcos, uno hacia distal y otro hacia palatino, formando un ángulo muy obtuso de ramas muy abiertas, que circunscribe a la cúspide distal. Esta es la cúspide más pequeña en tamaño. Las otras dos cúspides que se describen en la cara oclusal del PMSP son la mesiopalatina, que es la mayor en tamaño, y la distovestibular. Estas cúspides se encuentran unidas por una estructura de esmalte llamada apófisis oblicua.

Teniendo en cuenta estas características establecidas por Figún y Garino [5], se hace posible la diferenciación con otras piezas dentarias y, en particular, con el segundo molar superior permanente (SMSP) [6-8], que presenta caras oclusales muy semejantes, lo que dificulta su identificación. Se parte de la idea de que, en el segundo molar, se encuentran los mismos elementos arquitectónicos descritos en el primero, es decir, cuatro cúspides, dos fosas principales y cuatro surcos principales, y se agrega un quinto surco que se ubica sobre la apófisis adamantina y la interrumpe en su totalidad, y así une a la fosa central con la fosa distal. Por lo tanto, no se detecta la presencia de la apófisis oblicua, en el sMsp, pues en su lugar se encuentra un surco $[9,10]$.

Clínicamente puede afirmarse que para diferenciar desde su cara oclusal un PMSP de un SMSP, es necesario observar la presencia o ausencia de la apófisis oblicua. Si en toda su extensión no se identifica otro elemento arquitectónico, estamos en presencia de un primer molar y si en su lugar hay un surco, afirmaremos que es un segundo molar $[11,12]$.
Durante la enseñanza de la anatomía dentaria a estudiantes de la carrera de Odontología, específicamente en Introducción a la Odontología, el grupo de docentes observó que, en algunas ocasiones, al tratar de identificar y diferenciar estas piezas dentarias, aparecía en el PMSP un surco que, sin cortar totalmente a la apófisis oblicua, la recorría dificultando su reconocimiento. Es así que surge el estudio "Identificación de elementos anatómicos no tipificados en la cara oclusal del primer molar superior permanente", que ha permitido visualizar una nueva forma de presentación del PMSP no descrita en la literatura [13].

Se consideró Tipo I a la cara oclusal del PMSP, caracterizada por los anatomistas clásicos, y Tipo II a la cara oclusal del PMsP, con presencia de un surco que interrumpe parcialmente a la apófisis oblicua. Los resultados han arrojado que el $82 \%$ de las piezas observadas pertenece al Tipo II, identificado por el grupo de investigación $[12,14]$. Simultáneamente, y para confirmar estas afirmaciones, se desarrolló un trabajo cuyo propósito fue averiguar si se trataba de un surco o si, en realidad, era una fisura. Los datos arrojaron resultados compatibles con una entidad circunscrita en el esmalte dentario, lo que confirma que se trata de un surco y no de una fisura. Es por ello que, evidenciado el surco en la superficie oclusal del PMSP, surgió la necesidad de averiguar cuál era la dirección que adoptaba, dónde se originaba y cuál era su longitud [15], para poder registrarlo como una nueva presentación o variable anatómica de cara oclusal.

\section{Métodos}

El estudio fue observacional y descriptivo. En una primera etapa, se trabajó con 50 piezas inertes de la cátedra Introducción a la Odontología y con 128 PMSP naturales de pacientes que concurrieron al servicio de odontología del Hospital J. R. Vidal de la ciudad de Corrientes-Capital y que dieron voluntariamente su consentimiento para formar parte de la muestra. Los criterios de inclusión fueron: PMSP con corona completa, libres de restauración y patologías. Se excluyeron piezas dentarias con defectos de forma y tamaño, con caries activas o detenidas de fosas y fisuras, con restauraciones de la superficie oclusal y traumatismos que impedían la visualización de su superficie.

Las piezas dentarias inertes seleccionadas fueron acondicionadas mediante la limpieza de su superficie oclusal por medio de brochas en forma de copa de 
cerdas semiduras a baja velocidad, con pasta de piedra pómez y agua. Luego se procedió a enjuagar cada una de las piezas bajo chorro de agua corriente y mediante jeringa triple; se secaron enérgicamente con aire. Las observaciones se realizaron con lupa y luz natural. En los PMSP naturales de pacientes se hizo una detallada profilaxis para eliminar detritus para luego observarlas mediante la utilización de espejo bucal y luz de la unidad dental.

Los datos de la observación fueron registrados en planillas confeccionadas para tal fin, teniendo en cuenta los siguientes parámetros:

- Diente natural inerte: aquella pieza dentaria aislada, seca e inerte.

- Diente natural de pacientes: aquella pieza normalmente implantada (16 o 26) que clínicamente permite visualizar su corona en la cavidad bucal (corona clínica).

Las características anatómicas halladas se agruparon teniendo en cuenta la siguiente ponderación:

- Tipo I: cara romboidal con la configuración externa de la superficie oclusal descrita por Figún y Garino [2].

- Tipo II: cara romboidal con la configuración externa de la superficie oclusal más un surco que interrumpe parcialmente la apófisis oblicua.

Una vez recabada la información, se analizaron los datos obtenidos para expresar en forma numérica en qué proporción aparecen las características anatómicas Tipo I y Tipo II, en los primeros molares superiores.

El análisis estadístico se basó en el cálculo de la media. En una segunda etapa, y con la finalidad de obtener datos sobre el origen, dirección y longitud, se utilizaron 43 piezas dentarias inertes, obtenidas durante el periodo 2003-2010 y pertenecientes a la morfoteca de la cátedra Introducción a la Odontología, que presentaban las características Tipo iI y se encontraban libres de restauración o lesión, con corona completa. Se incluyeron además 20 piezas dentarias naturales normalmente implantadas en boca, que presentaron cara oclusal compatibles con el Tipo II (presencia de surco que interrumpe parcialmente la apófisis oblicua), libres de lesión o restauración, con corona completa, de pacientes de ambos sexos, de edades comprendidas entre los 18 y los 45 años, que concurrieron voluntariamente al Hospital Odontológico Universitario de la Facultad de Odontología de la unNe. Se trabajó así con un total de 63 piezas dentarias naturales inertes y naturales de pa- cientes que presentaron el surco no tipificado sobre la apófisis oblicua, reconocido como cara oclusal Tipo II (figuras 1 y 2).

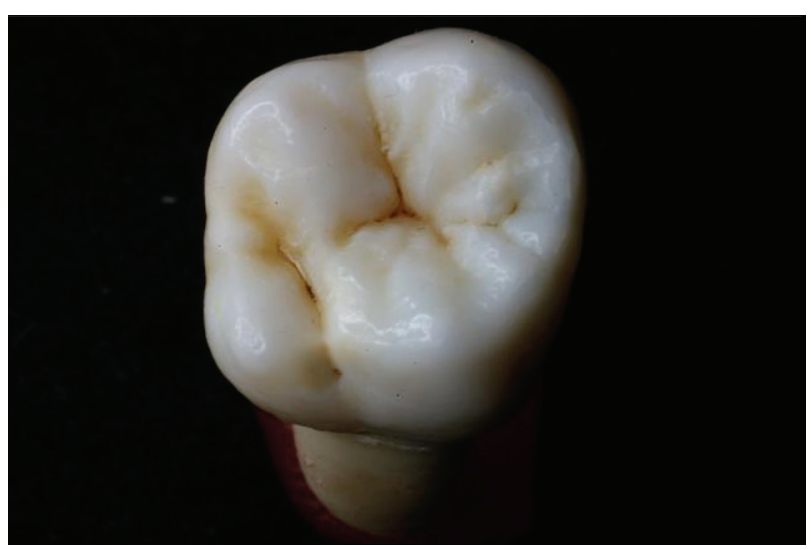

Figura 1. PMSP natural inerte Tipo II con surco *Observación directa de un PMSP natural inerte Tipo II Fuente: Cátedra Introducción a la Odontología

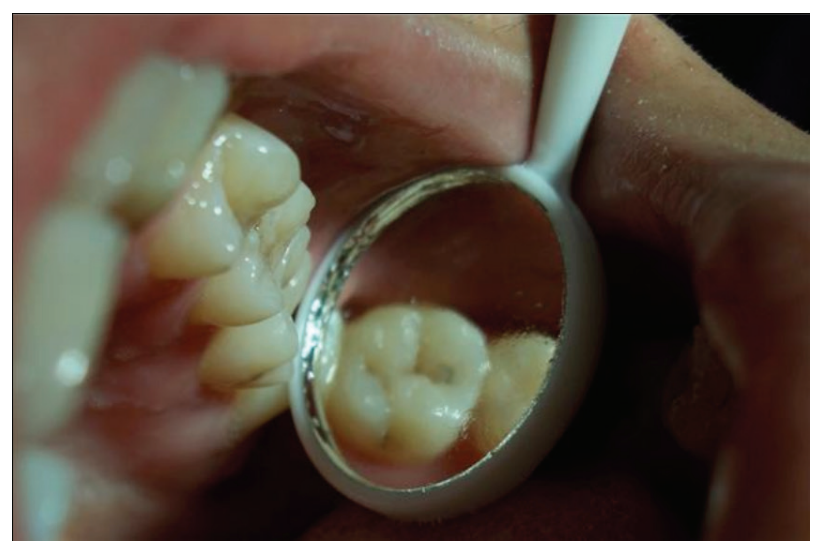

Figura 2. PMSP natural de paciente Tipo II con surco medio

*Método indirecto utilizado para la observación de la cara oclusal del PMSP Tipo II

Fuente: Cátedra Introducción a la Odontología

Seleccionadas las piezas dentarias inertes, fueron acondicionadas dejándolas sumergidas en una solución de hipoclorito de sodio al 5\% diluido en agua durante 15 minutos, luego se enjuagaron utilizando chorro de agua corriente y, por medio de un cepillo manual de cerdas semiduras, se procedió a retirar todo resto de material orgánico. Se colocaron en papel absorbente para secarlas. Mediante observación con lupa de mano, se observó el origen y la dirección del surco. Seguidamente, se procedió a medir la longitud utilizando un compás y trasladando esa medida a una regla milimetrada (figura 3). Los datos fueron volcados en planillas confeccionadas para tal fin. 


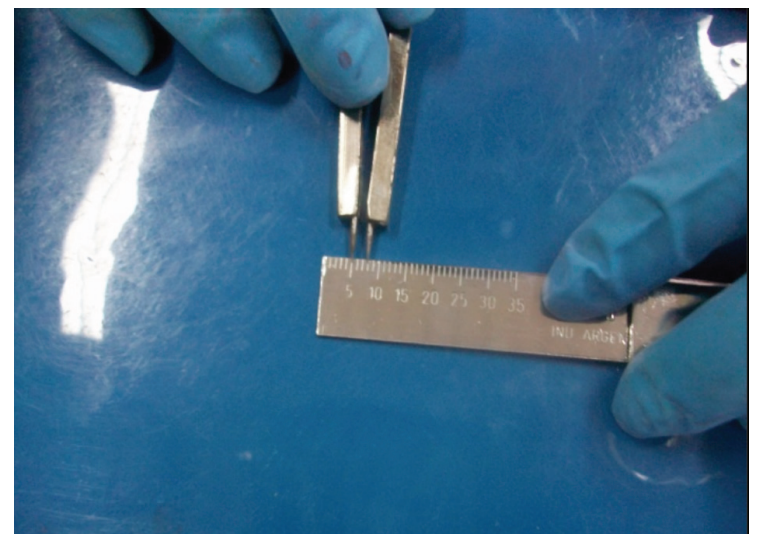

Figura 3. Registro de la longitud

${ }^{*}$ Regla metálica milimetrada utilizada para transportar las medidas obtenidas con el compás

Fuente: Cátedra Introducción a la Odontología

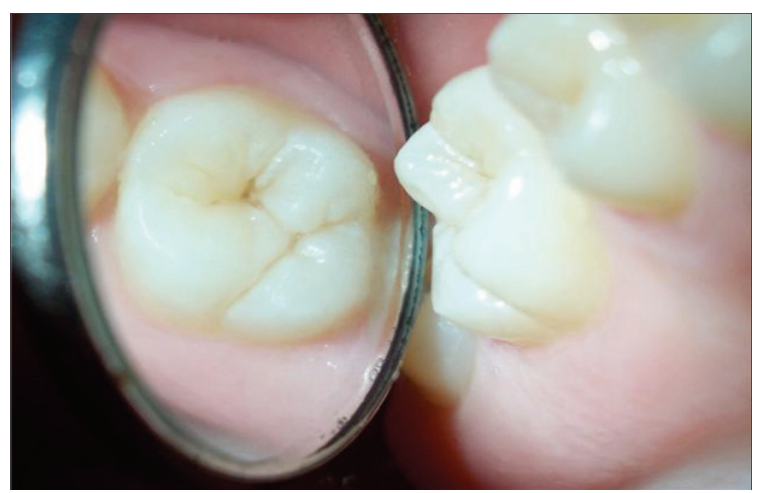

Figura 4. PMSP natural Tipo II observación indirecta * Método indirecto utilizado para la observación de la cara oclusal del PMsP Tipo II

Fuente: Cátedra Introducción a la Odontología

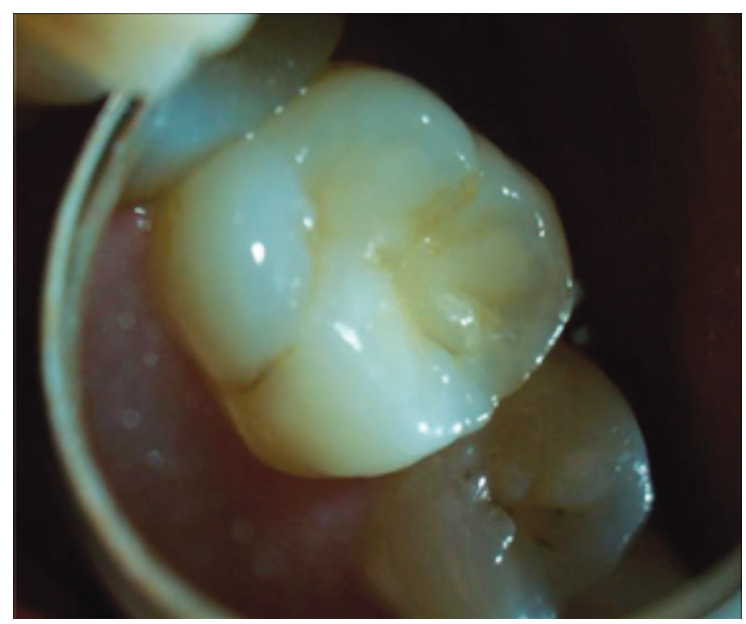

Figura 5. PMSP natural de paciente Tipo I

${ }^{*}$ Método indirecto utilizado para la observación de la cara oclusal del PMSP Tipo I

Fuente: Cátedra Introducción a la Odontología
A las piezas dentarias en boca se les realizó profilaxis con pasta y cepillado mecánico. Luego se procedió a la observación indirecta utilizando espejo bucal (figura 4) y al registro de datos en relación con el origen y dirección del surco. Para determinar la longitud, se tuvieron en cuenta los siguientes parámetros:

Se consideró surco corto (sc) a los que medían menos de $1 \mathrm{~mm}$, surco medio (sM) a los que se ubicaban entre 1 y $2 \mathrm{~mm}$, mientras que se consideró surco largo (sL) a los que obtenían una medida superior a $2 \mathrm{~mm}$. Para registrar la dirección del surco, se tuvieron en cuenta las siguientes posibilidades: dirección distovestibular, aquella que se inicia en la fosa central y se dirige hacia distal y vestibular; dirección distopalatina a la que sale de la fosa central y se dirige hacia distal y palatino; dirección distal, a la que sale de la fosa central y tiende a dirigirse netamente hacia distal; y dirección mesial considerando los casos en que el surco sale de la fosa distal y se dirige hacia mesial. Los datos obtenidos de cada una de las observaciones y mediciones realizadas tanto en dientes inertes como en pacientes fueron registrados en planillas y a través de fotografías digitales.

\section{Resultados}

Se realizó un análisis estadístico de tipo descriptivo. Sobre un total de 178 PMSP naturales e inertes observados (tablas 1 y 2; figuras 1, 2, 4 y 5), el 74\% presentó, en la superficie oclusal, un surco que interrumpía parcialmente la apófisis oblicua, reconocido como PMSP con cara oclusal Tipo II (figura 6).

Tabla 1. Tipos de caras oclusales en PMSP inertes

\begin{tabular}{|l|c|c|}
\hline Caras oclusales / Frecuencias & fa & fr \\
\hline Tipo I & 22 & $44 \%$ \\
\hline Tipo II & 28 & $56 \%$ \\
\hline Total & 50 & $100 \%$ \\
\hline
\end{tabular}

${ }^{\star}$ El $56 \%$ de las piezas dentarias inertes observadas ha presentado un surco que interrumpe parcialmente la apófisis oblicua

Fuente: datos de la observación directa en dientes inertes

Tabla 2. Tipos de caras oclusales en PMSP de pacientes

\begin{tabular}{|l|c|c|}
\hline \multicolumn{1}{|c|}{ Caras oclusales / Frecuencias } & fa & fr \\
\hline Tipo I & 25 & $19,53 \%$ \\
\hline Tipo II & 103 & $80,46 \%$ \\
\hline Total & 128 & $100 \%$ \\
\hline
\end{tabular}

${ }^{\star}$ El 80,46\% de las piezas dentarias naturales observadas ha presentado un surco que interrumpe parcialmente la apófisis oblicua

Fuente: datos de la observación indirecta en pacientes 


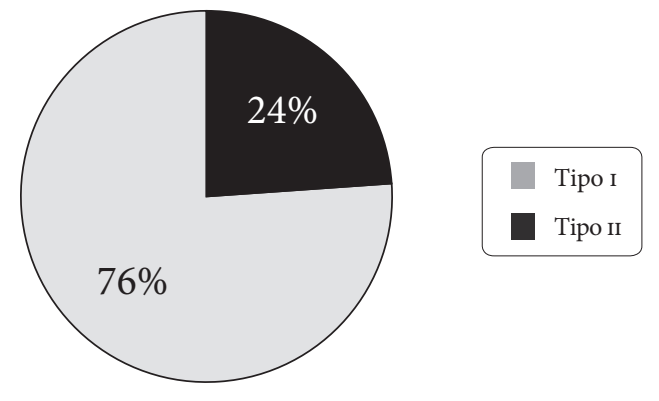

Figura 6. Determinación de PMSP inertes y naturales Tipo I y Tipo II sobre el total de la muestra

${ }^{\star}$ La figura muestra que el $76 \%$ de los PMSP observados presentaron el surco que los distingue como PMSP Tipo II

Fuente: datos obtenidos de las observaciones realizadas

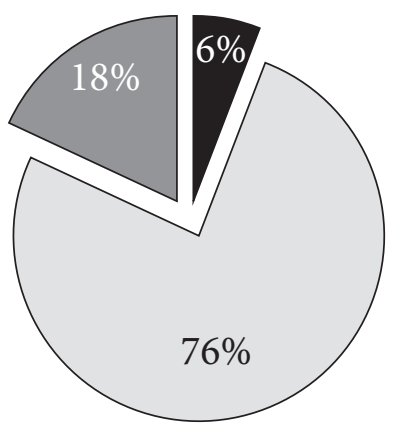

Surco corto

Surco medio

Surco largo

Figura 7. Longitud obtenida en la medición de los surcos

*Se puede visualizar que el $76 \%$ de los surcos presentaron una longitud media, entre 1 y $2 \mathrm{~mm}$

Fuente: datos obtenidos de las mediciones realizadas en los surcos de PMSP

Sobre un total de 63 PMSP Tipo II observados, los resultados obtenidos fueron: el $100 \%$ de los surcos presentó su origen en la fosa principal central triangular y una dirección oblicua hacia palatino y distal. Con respecto a la longitud, se registró un $76 \%$ de surcos medios (figura 7) con un promedio de $1,7 \mathrm{~mm}$. Le siguieron en orden los surcos largos de $2 \mathrm{~mm}$ o más y, por último, los cortos de entre 0 y $1 \mathrm{~mm}$ (tabla 3 , figura 8 ).

Tabla 3. Longitud, dirección y origen del surco en PMSP Tipo II

\begin{tabular}{|l|c|c|}
\hline \multicolumn{1}{|c|}{ Características/frecuencias } & fa & fr \\
\hline Longitud & & \\
\hline Corto hasta 1 mm & 4 & $6,34 \%$ \\
\hline Medio 1 a 2 mm & 48 & $76,19 \%$ \\
\hline Largo más de 2 mm & 11 & $17,4 \%$ \\
\hline Dirección & & \\
\hline Distopalatina & 63 & $100 \%$ \\
\hline Origen & & \\
\hline Fosa principal central & 63 & $100 \%$ \\
\hline
\end{tabular}

Fuente: datos obtenidos del estudio del surco
La tabla 3 muestra que el $76,19 \%$ de los PMSP Tipo II posee surco considerado medio, una dirección hacia distal y palatino y el surco nace en la fosa principal central de la cara oclusal.

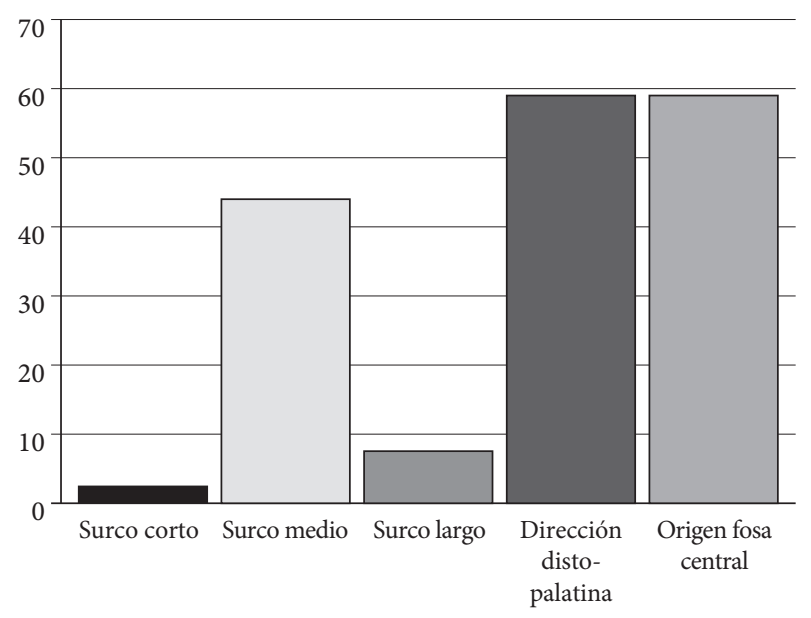

Figura 8. Longitud, dirección y origen de los surcos en relación con 63 PMSP Tipo II

Fuente: datos obtenidos mediante observación y medición de los surcos

La figura 8 muestra que 48 PMSP presentaron el surco con una longitud media; le siguieron en orden los surcos largos y cortos. La dirección que adoptó en todos los casos fue hacia distal y palatino. El 100\% se originó de la fosa principal central.

\section{Discusión}

Los anatomistas clásicos consideran al primer molar superior permanente con una sola presentación de cara oclusal caracterizada por presentar una forma romboidal [1]. Sobre la superficie oclusal evidencian la aparición de cuatro cúspides, dos fosas principales y cuatro surcos, resaltando la presencia de un elemento arquitectónico distintivo, la apófisis oblicua [2]. A su vez, los anatomistas Figún y Garino, en coincidencia con otros autores, consideran y describen una sola forma de presentación de cara oclusal en el primer molar superior permanente. Sin embargo, el trabajo realizado estaría poniendo en conocimiento una nueva forma de presentación de cara oclusal para el primer molar superior permanente, denominada Tipo II, que si bien mantiene la forma establecida por los autores, le adicionaría la presencia de un quinto surco con características propias, ubicado en la superficie oclusal y que atraviesa parcialmente a la apófisis oblicua, sin cortarla [3]. 


\section{Conclusiones}

El PMSP puede presentar dos tipos de cara oclusal: la romboidal con apófisis oblicua, descrita por Figún y Garino, llamada Tipo I por el grupo de investigación, y la romboidal con apófisis oblicua interrumpida parcialmente por un surco, llamada Tipo II.

Este surco se ha podido caracterizar mediante la observación y medición en piezas naturales inertes y piezas naturales normalmente implantadas en boca. $\mathrm{Su}$ longitud es media, varía entre 1 y $2 \mathrm{~mm}$, se origina en la fosa principal central de la cara oclusal y se dirige oblicuamente hacia distal y palatino, con diferentes inclinaciones. Este descubrimiento permitirá actualizar la morfología externa de la cara oclusal del PMSP, para su aplicación en la enseñanza de pregrado y posgrado. En la práctica clínica, su observación facilitará la detección precoz de lesiones, debido a que se trata de un elemento arquitectónico retentivo que cobra valor ante la necesidad de determinar zonas de riesgo de procesos cariosos.

Su conocimiento beneficiará la aplicación de técnicas de restauración y la devolución de la anatomía perdida.

\section{Referencias}

[1] Ash M, Stanley N. Anatomía, fisiología y oclusión dental. $8^{\mathrm{a}}$ ed. Madrid: Elsevier; 2004.

[2] Figún ME, Garino RR. Anatomía odontológica, funcional y aplicada. $2^{\text {a }}$ ed. Buenos Aires: El Ateneo; 2010.

[3] Diamond M. Anatomía dental. 2a ed. México: Uteha; 1992.

[4] Velayos J, Díaz H. Anatomía de la cabeza con enfoque odontoestomatológico. Madrid: Médica Panamericana; 1994.
[5] Aprile H, Figún ME, Garino RR. Anatomía odontológica orocervical. 5a ed. Buenos Aires: El Ateneo; 1984.

[6] Ash MS. Anatomía dental fisiología y oclusión. 5a ed. México: Interamericana; 1994.

[7] Diamond M. Anatomía dental con la anatomía de la cabeza y del cuello. México: Uteha; 1992.

[8] Kraus B, Jordan R, Abrams L. Anatomía dental y oclusión. México: Nueva Editorial Interamericana; 1972.

[9] Sicher H, Du Brul EL. Anatomía oral. Barcelona: Doyma; 1990.

[10] Brand R, Isselhard D. Anatomía de la estructuras orofaciales. Madrid: Harcourt Brace; 1999.

[11] Tortora GJ, Reynolds S. Principios de anatomía y fisiología. España: Mosby Dogma; 1996.

[12] Discacciati MS, Bessone GG, Mansutti FL. Determinación de las características anatómicas no tipificadas presentes en la apófisis oblicua del primer molar superior permanente. Corrientes. 2010;3(2):15-20.

[13] Bessone GG, González MM, Guiglioni MA. Presencia de surco no tipificado sobre apófisis oblicua del primer molar superior. Revista de la Facultad de Odontología, Universidad Central del Ecuador. 2009;11:53-6.

[14] Bessone GG, Guiglioni MA, González MM. Presentación anatómica en dientes inertes del surco presente en la cara oclusal del primer molar superior permanente [internet]. 2012 [citado 2013 jun 25]. Disponible en: http://www.unne.edu.ar/unnevieja/investigacion/ com2012/CM-096.pdf

[15] Discacciati MS, Pared MG, Bessone GG. Determinación de la longitud del surco presente en la apófisis oblicua del primer molar superior permanente. Corrientes. 2012;1:15-22. 